\title{
KOMPASS
}

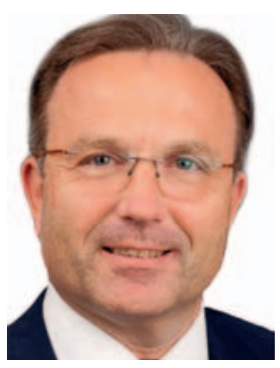

Markus Möhler

Universitätsmedizin Mainz I,

Medizinische Klinik und Poliklinik,

Mainz, Deutschland

Die aktuelle Ausgabe des Karger Kompass OnKologie widmet sich den klinisch zentralen Diskussionen zur Therapie des fortgeschrittenen oder noch nicht diffus metastasierten Magenkarzinoms. Mit dem klar etablierten Standard der perioperativen Therapie haben wir seit ca. 8 Jahren zahlreiche Patienten gegenüber den ursprünglich alleinigen Operation zusätzlich geheilt. Da häufig bereits Mikrometastasen vorliegen, die der üblichen Diagnostik entgehen, entscheidet sich gerade hier, ob und wie weitere Patienten durch neue innovative Therapieansätze geheilt werden können. Ist die perioperative Therapie auch bei älteren Patienten sinnvoll? Dieser Frage widmet sich Kollege Stahl aus Essen mit einem gelungenen Kommentar zur Kohortenanalyse von Herrn Haag und Kollegen aus Heidelberg [1]. Hier hatten die neoadjuvant behandelten Patienten Oxaliplatin-haltige Protokolle bekommen, um die Nebenwirkungen von Cisplatin zu vermeiden. 80\% der Patienten wurden zudem operiert. Während bei jüngeren Patienten etwa 50\% postoperativ die adjuvante Therapie fortsetzten, war dies bei älteren jedoch nur in etwas mehr als einem Viertel der Fälle möglich.

Frau Kollegin Rau aus Berlin berichtet in ihrer Übersichtsarbeit über die ersten Erfolge und weiteren Herausforderungen der Therapie von peritonealen Oligometastasen gastrointestinaler Tumoren [2]. Der multimodale Ansatz mit gezielter Chirurgie,

\section{Das lokal fortgeschrittene Magenkarzinom - eine therapeutische Herausforderung}

Chemotherapie direkt in den Bauchraum und Hyperthermie (HIPEC) wurde von Protagonisten wie Sugarbaker, Glehen und Elias etabliert und propagiert. Hier sind die komplette Zytoreduktion (CCR) und das Ausmaß der peritonealen Streuung als Peritonealkarzinoseindex (peritoneal carcinomatosis index, P(I) entscheidend, um die Chance auf langfristige Tumorfreiheit zu bestimmen. Inwieweit die Hyperthermie zusätzlich zur intensiven peritonealen Chirurgie mit intraabdomineller Chemotherapie sinnvoll ist, konnte bisher noch nicht eindeutig belegt werden. Leider gab und gibt es bisher zu wenige gute Studien. Frau Rau führt daher zu Recht die große deutsche GASTRIPEC-Studie durch, die die Wertigkeit der Hyperthermie randomisiert bestimmt. Darüber hinaus sind leider auch die intra- peritoneal genutzten Chemotherapieprotokolle veraltet und neue innovative gezielte Therapien oder Antikörper bisher nicht kombiniert analysiert worden. Weitere Analysen der Häufigkeit von Peritonealkarzinosen und dem Therapieansprechen nach den neuen Subgruppen in den genomischen Atlanten (TGCA) von Magen- und Darmkrebs stehen ebenfalls aus.

Frau Kollegin Hadzitheodorou aus New Jersey diskutiert an 4 Patienten erneut die Frage, ob eine adjuvante Radiochemotherapie nach neoadjuvanter Chemotherapie und Operation sinnvoll ist [3]. Während ihrer Publikation erschien zeitgleich das Ergebnis der CRITICS-Studie, die keinen Vorteil einer adjuvanten Radiochemotherapie gegenüber einer adjuvanten Chemotherapie feststellen konnte [4].
Abb. 1. Aktuelle Studien der AIO und EORTC zur Therapie des lokal fortgeschrittenen Magenkrebses.

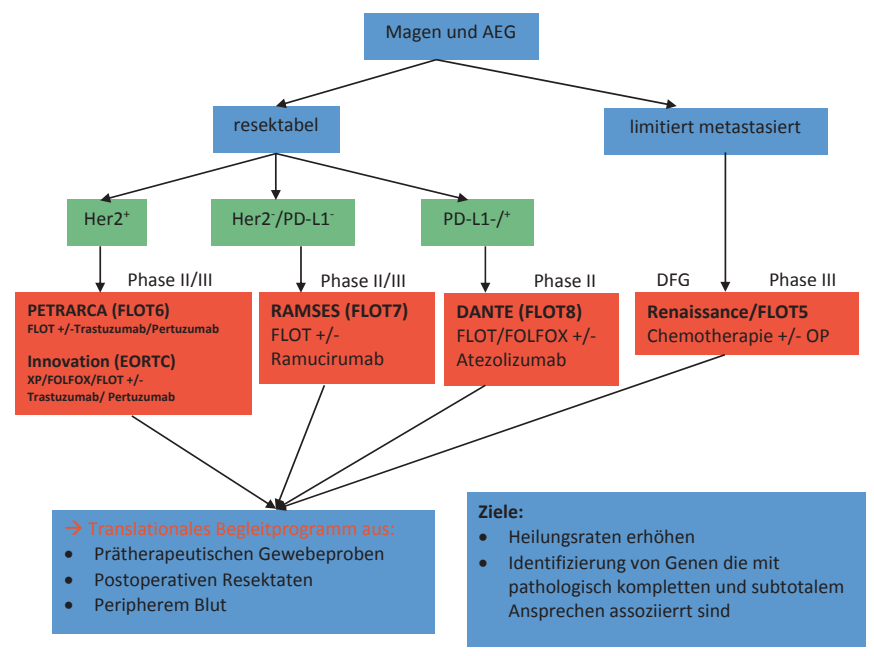

\section{KARGER}

Fax +497614520714

information@karger.com

www.karger.com (c) 2017 S. Karger GmbH, Freiburg

Accessible online at: www.karger.com/kko
Prof. Dr. Markus Möhler

Universitätsmedizin Mainz

Medizinische Klinik und Poliklinik

Langenbeckstraße 1, 55131 Mainz, Deutschland

Markus.Moehler@unimedizin-mainz.de 
Die beiden laufenden Studien TOPGEAR aus Australien/Europa und ARTIST2 aus Asien stehen mit ihren Ergebnissen zu dieser Frage noch aus [5].

Wenn wir diese Publikationen mit der 2011 publizierten deutschen S3-Leitlinie zu Magenkrebs [6] vergleichen, so wird klar, dass diese bereits früher Stellung bezogen und noch ihre Gültigkeit hat (Abb. 1). Dennoch ist es höchste Zeit, diese Standards wieder neu zu überprüfen und besser zu definieren, auch um qualitätsgesicherte Zentren für solche multimodale Therapien zu stärken. Für die aktiven Fachgesellschaften in Diagnostik und Therapie des Magenkrebses haben wir uns daher als Experten erneut zusammengetan, um bis zum deutschen Krebskongress (DKK) 2018 ein sorgfältiges S3-Leitlinien-Update zu erstellen. Mit den definierten Methoden der evidenzbasierten Medizin widmen wir uns den neuen genetischen Markern, interdisziplinären Konzepten zur Oligometastasierung und Therapieempfehlungen für ältere Patienten. Die Arbeitsgemeinschaft internistischer Onkologie (AIO) und die europäische Studiengruppe EORTC haben in Kooperation mit Chirurgen und Strahlentherapeuten wertvolle perioperative Projekte aufgelegt. Es ist zu wünschen, dass in diese Konzepte gut rekrutiert wird, nicht nur, um die Chance auf individuelle Heilung zu verbessern, sondern vor allem, um valide statistische Grundlagen mit neuen Standards zu legen. Auch über den DKK im Februar 2018 hinaus ist die Teilnahme an zu diesem Thema laufenden Studien, wie z.B. auch von Frau Rau in Berlin, wissenschaftlich sehr willkommen und zu empfehlen.
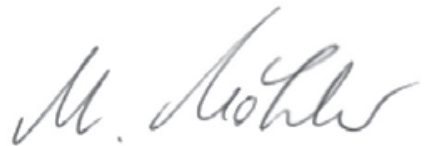

Prof. Dr. Markus Moehler

\section{Literatur}

1 Stahl M: Perioperative Chemotherapie beim Magenkarzinom: Ältere Patienten können ebenso profitieren wie jüngere. Karger Kompass Onkol 2017;4:80-81.

2 Rau B, Brandl A, Pascher A, et al.: Oligometastatische Erkrankung in der Peritonealhöhle bei gastrointestinalen Karzinomen. Karger Kompass Onkol 2017;4:73-78.

3 Hadzitheodorou C, Moss RA, Kennedy TJ, Jabbour SK: Neoadjuvante Chemotherapie und adjuvante Radiochemotherapie in der Behandlung des resezierten Adenokarzinoms des Magens: Eine Fallserie. Karger Kompass Onkol 2017;4:113-117.

4 Castellino A: CRITICS study: equal benefit from adjuvant CT, CRT in resectable gastric cancers. am.asco.org/critics-study-equal-benefit-adjuvant-ct-crtresectable-gastric-cancers-0 (letzter Abruf: 20.09.2017).

5 Lee J, Lim DH, Kim S, et al.: Phase III trial comparing capecitabine plus cisplatin versus capecitabine plus cisplatin with concurrent capecitabine radiotherapy in completely resected gastric cancer with D2 lymph node dissection: the ARTIST trial. J Clin Oncol 2012;30:268-273.

6 Leitlinienprogramm Onkologie: Magenkarzinom. Diagnostik und Therapie der Adenokarzinome des Magens und ösophagogastralen Übergangs. www.leitlinienprogramm-onkologie.de/leitlinien/magenkarzinom (letzter Abruf: 19.09.2017). 


\section{FOKUS}

\section{Magenkarzinom}

\section{(7i) Epidemiologie}

Trotz sinkender Neuerkrankungs- und Sterberaten erkranken weltweit jedes Jahr etwa 1 Mio. Menschen an Magenkrebs, auf den nach Lungen- und Leberkrebs auch die meisten Krebstodesfälle entfallen. Im Jahr 2013 erkrankten in Deutschland 15631 Menschen an Magenkrebs - etwa halb so viele wie noch 40 Jahre zuvor. Die Zahl der Sterbefälle ist in diesem Zeitraum sogar auf etwa ein Drittel (9622 Fälle) zurückgegangen - und das, obwohl das Erkrankungsrisiko mit dem Alter ansteigt und die Anzahl älterer Menschen in der Bevölkerung deutlich zugenommen hat. Männer erkranken weiterhin häufiger als Frauen, etwa im Verhältnis 3:2.

Histologisch überwiegen im Magen Adenokarzinome und seltener spezielle Formen, die zum Teil vor allem dort vorkommen, wie Siegelzellkarzinome (15\%). Eine Besonderheit stellen von der Magenschleimhaut ausgehende (Mucosa-assoziierte) MALT-Lymphome dar, die zu den niedrig malignen Non-Hodgkin-Lymphomen gerechnet werden. Neben Karzinomen kommen selten mesenchymale und Mischtumoren oder neuroendokrine Karzinome vor.

Sowohl in Deutschland als auch weltweit gehört Magenkrebs zu den Krebsarten mit der derzeit am stärksten sinkenden altersstandardisierten Sterblichkeit. Seit mehr als 10 Jahren gehen die Sterberaten durchschnittlich um etwas mehr als 3\% pro Jahr zurück. Dieser Rückgang hält weiter an und ist vermutlich auf verbesserte Lebens- und Ernährungsgewohnheiten zurückzuführen. Internationale und regionale Vergleiche der Sterbe- und Erkrankungsraten zeigen ein West-Ost-Gefälle in Europa und innerhalb Deutschlands. Allerdings steigt die Anzahl der Karzinome des oesophagogastralen Übergangs (AEG) und des unteren Oesophagus an. Die Überlebensaussichten mit Magenkrebs haben sich in letzter Zeit zwar verbessert, im Vergleich mit anderen Krebserkrankungen sind sie jedoch weiterhin eher ungünstig. Immer noch stirbt 1\% der Bevölkerung an Magenkrebs, die relativen 5-Jahres-Überlebensraten liegen knapp über 30\%. Das mittlere Erkrankungsalter liegt mit 72 Jahren bei Männern und 75 Jahren bei Frauen vergleichsweise hoch. Etwa zwei Drittel aller Erkrankungen wird erst in einem fortgeschrittenen Stadium (T3-T4) entdeckt.

\section{Herausforderungen und Handlungsbedarf}

Eine bakterielle Infektion des Magens mit Helicobacter pylori (H. pylori) ist der wichtigste Risikofaktor für Magenkrebs. Die H. pylori-Eradikation mit dem Ziel der Magenkarzinomprophylaxe kann bei Risikopersonen durchgeführt werden. Wichtige Risikofaktoren für das distale Magenkarzinom sind Alter, niedriger sozio-ökonomischer Status, Tabakrauchen, Alkoholkonsum, familiäre Belastung, Übergewicht, vorangegangene Magenoperationen, perniziöse Anämie, Leben in einer Hochrisikopopulation sowie Ernährungs- und Umweltfaktoren.

Neben dem neuen humanen Krebsgenom-Atlas (TCGA) mit 4 prognostisch unterschiedlichen Krebs-Subtypen rücken vererbte Karzinome wie die E-cadherin-Mutation (CDH-1-Gen) oder die HNPCC-Karzinome bei jüngeren Patienten in den Vordergrund: Denn Verwandte ersten Grades von Patienten mit einem Magenkarzinom haben ein im Vergleich zur Normalbevölkerung erhöhtes Risiko, ebenfalls an einem Magenkarzinom zu erkranken. Ein frühes Erkrankungsalter und vor allem das Vorlie- gen eines diffusen Magenkarzinoms (oder Siegelring-Typs) und eine familiäre Häufung sind Hinweise auf das Vorliegen eines hereditären diffusen Magenkarzinoms. In diesem Fall sollte eine genetische Beratung der Familie erfolgen. Bei Nachweis einer Keimbahnmutation des $\mathrm{CDH}-1$ Gens sollte Patienten ab dem 20. Lebensjahr eine prophylaktische Gastrektomie angeboten werden. Auch Patienten mit hereditärem kolorektalem Karzinom ohne Polyposis (HNPCC, Lynch-Syndrom) sollten über ihr erhöhtes Lebenszeitrisiko für die Entwicklung eines Magenkarzinoms aufgeklärt werden und eine Gastroskopie angeboten bekommen.

\section{(1) Prävention}

Die Entwicklung eines zuverlässigen Impfstoffs gegen H. pylori-Infektionen steht noch aus. Die frühzeitige Entdeckung und Behandlung einer chronischen Infektion mit H. pylori bietet jedoch das Potential, die Krankheitslast aufgrund von Magenkrebs zu senken.

In Deutschland kann nach aktuellen Leitlinien bei Personen mit erhöhtem Risiko und Nachweis einer Infektion eine antibiotische Kombinationstherapie (Eradikationstherapie) durchgeführt werden, um das Magenkrebsrisiko zu senken. Die fokale Atrophie und intestinale Metaplasie sind hier ebenfalls mit H. pylori ein Risikofaktor.

Ein serologisches Screening der asymptomatischen Normalbevölkerung sollte jedoch nicht durchgeführt werden. Außerdem sollten ASS oder NSAR nicht zur Prophylaxe des Magenkarzinoms angewendet werden. Gleichzeitig gibt es keine gesicherten Hinweise, dass die dauerhafte Einnahme von Protonenpumpeninhibitoren (PPI) das Risiko für ein Magenkarzinom erhöhen.

\section{Literatur}

Zentrum für Krebsregisterdaten (ZfKD) im Robert Koch-Institut: Magenkrebs (Magenkarzinom). Stand 17.12.2015. www.krebsdaten.de/Krebs/DE/Content/ Krebsarten/Magenkrebs/magenkrebs_node.html (letzter Zugriff 08.08.2017).

Zentrum für Krebsregisterdaten (ZfKD) im Robert Koch-Institut: Thema des Monats August 2017 - Das Risiko für Magenkrebs sinkt - nicht nur in Deutschland. Stand 01.08.2017. www.krebsdaten.de/Krebs/DE/Content/ Publikationen/Thema_des_Monats/Thema_des_Monats_node.html (letzter Zugriff 08.08.2017).

The Cancer Genome Atlas (TCGA), a collaboration between the National Cancer Institute (NCI) and National Human Genome Research Institute (NHGRI). www.cancergenome.nih.gov (letzter Zugriff 05.10.2017).

Deutsche Gesellschaft für Gastroenterologie, Verdauungs- und Stoffwechselkrankheiten (DGVS): Helicobacter pylori und gastroduodenale Ulkuskrankheit. www.dgvs.de/wissen-kompakt/leitlinien/leitlinien-der-dgvs/ helicobacter-pylori (letzter Zugriff 20.09.2017).

Leitlinienprogramm Onkologie: S3 Leitlinie Magenkarzinom - Diagnostik und Therapie der Adenokarzinome des Magens und ösophagogastralen Übergangs. www.awmf.org/leitlinien (letzter Zugriff: 08.08.2017). Leitlinie wird z.Zt. überarbeitet, Neufassung voraussichtlich Februar 2018.

Prof. Dr. Markus Möhler, I. Medizinische Klinik und Poliklinik, Universitätsmedizin Mainz 